\title{
Diferentes Magnitudes do Custo da Resposta em Um Múltiplo FI FI, Após Uma História em Um Múltiplo FR FR ${ }^{1}$
}

\author{
Carlos Eduardo Costa ${ }^{* a}$, João Paulo Pereira de Souza ${ }^{\text {a }}$, Thais Bianchini, \\ Tatiany Honório Porto ${ }^{\mathrm{b}}$ \& Luiz Alexandre Barbosa de Freitas ${ }^{\mathrm{c}}$ \\ ${ }^{a}$ Universidade Estadual de Londrina, Londrina, Brasil, ${ }^{\mathrm{b}}$ Universidade de São Paulo, São Paulo, Brasil \& \\ ${ }^{\mathrm{c}}$ Universidade Federal do Mato Grosso, Cuiabá, Brasil
}

\begin{abstract}
RESUMO
O estudo investigou (1) se a mudança de um múltiplo FR FR para um FI-custo FI-custo produziria diminuição na taxa de respostas e (2) o papel da magnitude do custo e sua ordem de apresentação. Participaram nove universitários expostos a um múltiplo FR60 FR60 até que o responder estabilizasse. Na Fase 2 distribuíram-se em dois grupos que variavam em relação a ordem de apresentação dos componentes: G1 - múltiplo FI custo 1 FI custo 10 e G2 - múltiplo FI custo 10 FI custo 1 . Todos os participantes emitiram altas taxas de respostas na Fase 1 e diminuíram as taxas na Fase 2.

Palavras-chave: custo da resposta; história comportamental; magnitude do custo; esquema de reforço; humanos.
\end{abstract}

\begin{abstract}
Different Response-cost Magnitudes on a Multiple FI FI After a Multiple FR FR History

This study investigated (1) if the transition from a multiple FR FR to a multiple FI-cost FI-cost would lower response rates and (2) the effects of different cost magnitudes and their order of presentation. Nine undergraduates were exposed to a multiple FR 60 FR 60 in Phase 1 until responding was stable. In Phase 2 they were distributed into two groups: G1 - multiple FI-cost 1 FI-cost 10 and G2 multiple FI-cost 10 FI-cost 1. For all participants, response rates decrease from Phase 1 to Phase 2 were high and low, respectively, in Phases 1 and 2.
\end{abstract}

Keywords: response cost; behavioral history; magnitude of the cost; schedules of reinforcement; humans.

Para entender melhor os efeitos da história no comportamento atual, pesquisadores têm criado histórias experimentais cujos efeitos são testados sobre as contingências atuais (Wanchisen, 1990). Uma questão importante sobre efeitos da história é sua duração, i.e., por quanto tempo o comportamento selecionado persiste depois de alterada a contingência de reforço (Costa, Cirino, Cançado, \& Soares, 2009; Lattal \& Neef, 1996). Por exemplo, os resultados de Weiner $(1965,1969,1970)$ sugeriram que após história de exposição a programas de reforço em razão, altas taxas de respostas persistiram durante todas as 10 sessões (com duração de 1 hora cada), mesmo quando esse padrão comportamental produzia perda de pontos.
Weiner (1965, Experimento 1) distribuiu seis participantes em três grupos com histórias de reforço distintas: razão fixa (FR), intervalo fixo (FI) ou reforço diferencial de baixas taxas (DRL). Após 10 sessões de uma hora, cada participante foi exposto a um FI 10 s-custo, em que recebia 100 pontos para a primeira resposta após 10 segundos e perdia um ponto para cada resposta emitida na sessão. Em cada programa de reforço, uma cor distinta de luz estava presente no painel com o qual os participantes interagiam. Durante o FR e o FI as taxas de respostas foram mais altas do que no DRL. Sob o FI-custo, os participantes expostos anteriormente ao FI ou ao DRL emitiram taxas de respostas mais baixas, do que os participantes com

Endereço para correspondência: Carlos Eduardo Costa - caecosta@uel.br 
história de FR. Ou seja, mesmo com a adição do custo ao programa de FI as altas taxas de resposta dos participantes com história em FR não diminuíram, sugerindo um efeito robusto da história comportamental.

Essa persistência comportamental em FI-custo, após uma história de responder em programas de razão, foi replicada em outros estudos. Por exemplo, Weiner (1969, Experimento 2) expôs os participantes inicialmente a um programa de FR, de DRL ou de DRL seguido por FR. Alguns participantes foram subsequentemente expostos a um FI-custo. Altas taxas de respostas ocorreram durante o FR, baixas taxas durante o DRL e, para os participantes expostos aos programas de DRL seguido de FR, foram observadas baixas e altas taxas de respostas, respectivamente. Sob o FI-custo, observou-se altas taxas de respostas para os participantes com história em FR e baixas taxas de respostas para os participantes com exposição prévia a DRL ou a DRL seguido de FR. Em suma, a taxa de respostas não diminuiu sob o FI-custo quando houve exposição anterior apenas ao FR, mas quando o FR foi precedido por exposição ao DRL, a taxa de respostas diminuiu durante a exposição ao FI-custo.

Outra replicação desse resultado foi o estudo de Weiner (1969, Experimento 5), que indicou que, independente do participante ser exposto a programas de razão fixa ou variável (VR) e do número de respostas exigidas para o reforço ser liberado ser 1, 20 ou 40, houve persistência de altas taxas de respostas quando o programa de reforço foi alterado para FI e FI-custo. A manutenção da taxa de respostas sob FI-custo, após uma história de responder em programas de razão, nos estudos de Weiner pode ter ocorrido porque a magnitude do custo (perda de um ponto) era baixa em relação à magnitude do reforçador (ganho de 100 pontos). Essa hipótese é sustentada pelos resultados de alguns estudos que sugerem que, quando estímulos aversivos são sobrepostos a contingências de reforço positivo, a supressão na taxa de respostas é diretamente proporcional à magnitude do estímulo aversivo (e.g., Azrin, 1960; Azrin \& Holz, 1961; Todorov, 1971).

Para avaliar a questão da magnitude do custo, Costa, Soares, Becker e Banaco (2009) investigaram os efeitos de diferentes magnitudes do custo sobre as taxas de respostas em um programa FI-custo, com participantes que haviam sido expostos a uma história em FR. Os participantes foram pagos pelos pontos obtidos na sessão (Grupo 1) ou por sessão, independente dos pontos obtidos (Grupo 2). Os participantes foram ex- postos a quatro fases compostas de três sessões com a duração de 15 minutos cada. Para todos os participantes, nas Fases 1 e 3, estava em vigor um FR 60 e, nas Fases 2 e 4, estiveram em vigor um FI 15 s-custo 1 e FI 15 s-custo 10, respectivamente. Os participantes de ambos os grupos emitiram taxas de respostas altas durante o FR (Fases 1 e 3 ) e quatro de cinco participantes de cada grupo emitiram taxas de respostas baixas em FI-custo 1. Quando o custo foi de 10 pontos, houve queda nas taxas de respostas para todos os participantes. Estes resultados não replicaram os de Weiner $(1965,1969,1970)$, pois as taxas de respostas da maioria dos participantes diminuíram no FI, mesmo quando o custo era de um ponto e esse resultado independeu de os pontos da sessão serem trocados ou não por dinheiro.

O estudo de Costa et al. (2009) teve algumas limitações experimentais que o presente estudo buscou solucionar. Costa et al. expuseram os participantes primeiro ao FI-custo 1 e, depois, ao FI-custo 10. É possível que a diminuição na taxa de respostas no FIcusto 10 tenha sido influenciada pela exposição precedente ao FI-custo 1. Na tentativa de sanar essas limitações o presente estudo utilizou um esquema múltiplo, que permite uma comparação paralela entre as condições (de custo 1 e custo 10) em vez de sequencial (cf. Freeman \& Lattal, 1992), no qual o valor do custo ( 1 ou 10) foi alternado entre os componentes de FI na fase de teste e a ordem de apresentação dos componentes foi controlada entre participantes. Essa comparação paralela pode contribuir para a validade interna do experimento, uma vez que possíveis variáveis estranhas também afetariam os resultados paralelamente (i.e., similarmente entre componentes).

Além disso, o estudo de Costa et al. (2009), por utilizar um número fixo de sessões em cada fase, não controlou a estabilidade da taxa de respostas em FR antes da exposição ao FI-custo. Segundo Joyce e Chase (1990), a probabilidade de mudança do comportamento quando as contingências mudam pode ser função da estabilidade do responder na linha de base. Outra característica do procedimento do presente estudo foi conduzir a fase de construção da história até a estabilidade das taxas de respostas.

Finalmente, nem o estudo de Costa et al. (2009) nem os estudos de Weiner $(1965,1969,1970)$ controlaram a taxa de reforço entre as duas fases do estudo. Okouchi (2003) sugeriu que a diferença no intervalo entre reforços (IRI) pode servir de controle de estímulos 
e, assim, poderia afetar a probabilidade de mudança ou persistência na taxa de respostas entre duas fases de um estudo.

Portanto, o objetivo do presente estudo foi investigar o efeito (1) da mudança de um múltiplo FR FR para um múltiplo FI-custo FI-custo sobre a taxa de respostas e (2) se a magnitude do custo em FI (i.e., perda de 1 ou 10 pontos) e a ordem de apresentação da contingência de custo (i.e., múltiplo FI-custo 1 FIcusto 10 ou múltiplo FI-custo 10 FI-custo 1) afetaria a velocidade e a magnitude da mudança na taxa de respostas.

\section{MÉTODO}

\section{Participantes}

Participaram nove universitários, de ambos os sexos (cinco homens e quatro mulheres), com faixa etária entre 19 e 24 anos (média de 21 anos). Nenhum participante era estudante de Psicologia ou tinha diagnóstico ou queixa de Lesão por Esforço Repetitivo.

\section{Equipamentos, Instrumento e Local}

As coletas de dados ocorreram em duas salas de aproximadamente $3 \mathrm{~m}^{2}$ cada. Cada sala continha um computador do tipo PC, com monitor em cores de 14 polegadas, mouse e teclado padrões, colocados sobre uma mesa; uma cadeira; um ventilador; um fone de ouvido e uma filmadora com tripé. O software utilizado na coleta de dados foi o ProgRef v3.1. (Costa \& Banaco, 2002, 2003) e para o cálculo da estabilidade foi utilizado o Stability Check (Costa \& Cançado, 2012).

\section{PROCEDIMENTO}

Antes da primeira sessão, cada participante leu e assinou um Termo de Consentimento Livre e Esclarecido (TCLE) que informava que o objetivo da pesquisa era "estudar algumas variáveis que possam afetar o modo como as pessoas se comportam em determinadas situações"; que seriam realizadas aproximadamente 20 sessões $^{2}$; a duração das sessões; que a tarefa experimental seria "ganhar o maior número de pontos possíveis (que aparecerão na tela do computador)"; que o participante usaria um fone de ouvido durante as sessões do qual soaria um "ruído branco ('chiado')" para isolamento acústico; que as sessões seriam fil- madas; que receberia $\mathrm{R} \$ 0,03$ para cada 100 pontos ganhos na sessão e que poderia abandonar a pesquisa a qualquer momento sem prejuízos.

Era solicitado aos participantes que deixassem fora da sala todo seu material, inclusive relógio, e que desligassem o telefone celular caso portassem algum. Em seguida, era entregue uma folha impressa com as seguintes instruções:

Esse trabalho não se trata de uma pesquisa sobre inteligência ou personalidade. Seu objetivo será ganhar pontos utilizando apenas o mouse. Os pontos aparecerão em uma janela (contador) que se localizará na parte superior da tela do computador na posição central. O experimentador não está autorizado a dar qualquer informação adicional. Caso haja dúvidas, releia estas instruções e prossiga o experimento. Bom trabalho!

Após a leitura da instrução, pelo participante, era solicitado que ele colocasse os fones de ouvido e a filmadora era ligada pelo experimentador.

Descrição geral da tarefa experimental: Inicialmente, a tela do computador - rodando o software ProgRef v3.1. - consistia em um fundo cinza e continha um botão na posição central superior com a palavra "Iniciar". Ao clicar nesse botão, a sessão era iniciada com a apresentação de outra tela que consistia em um fundo cinza, contendo um retângulo no centro inferior (botão de respostas), um contador de pontos no centro superior e um retângulo no canto superior direito (botão de resposta de consumação). A tarefa experimental consistia em clicar com o cursor do mouse sobre o botão de respostas. Quando a exigência de um programa de reforço era cumprida, aparecia no canto superior direito da tela um ícone "smile". O participante deveria clicar com o cursor sobre o botão de resposta de consumação. Ao fazer isso, o smile desaparecia e 100 pontos eram adicionados ao contador. Cada vez que o participante clicava sobre qualquer um dos botões, eles se movimentavam, simulando a pressão de um botão "físico", mas nenhum outro feedback da pressão ao botão (e.g., som) era fornecido.

Em um programa de reforço em FI o intervalo tinha início após o aparecimento do smile e não após a emissão da resposta de consumação. Portanto, o tempo gasto pelo participante para deslocar o cursor do mouse do botão de respostas ao botão de respostas de consumação e voltar para o botão de respostas era computado como parte do intervalo do FI e, com isso, 
o tempo gasto para emissão da resposta de consumação não afetava o intervalo entre reforços (IRI). Essa programação da resposta de consumação é semelhante à utilizada em algumas pesquisas com ratos em que água ou alimentos são utilizados como evento consequente, i.e., uma dada pressão à barra aciona o bebedouro ou libera uma pelota de comida, um novo intervalo é iniciado e o tempo que o rato passa lambendo o bebedouro ou comendo a pelota de alimento está dentro do intervalo do FI (sobre a importância da resposta de consumação em estudos com humanos ver, por exemplo, Matthews, Shimoff, Catania \& Sagvolden, 1977).

Quando o custo da resposta estava em vigor, um ou 10 pontos eram subtraídos do contador para cada resposta emitida pelo participante (exceto aquela que cumpria a exigência do programa de reforço em vigor). Tanto nos programas de FR quanto nos de FI, quando a exigência fosse cumprida mais de uma vez, sem que o participante clicasse no botão de resposta de consumação, outro smile não aparecia e o participante deixava de ganhar mais pontos enquanto não emitisse a resposta de consumação (i.e., não havia "acúmulo" de smiles). A ausência de emissão de uma resposta de consumação, que implicou em pontos não acrescentados ao contador, ocorreu de uma a nove vezes (mediana e moda de duas vezes) em toda Fase 1 para cada participante (exceto P2 e P5 que sempre emitiram a resposta de consumação) e nenhuma vez durante a Fase 2.

A Tabela 1 resume as fases do procedimento experimental.

Tabela 1

\begin{tabular}{cccccccccc} 
Resumo do Procedimento & \multicolumn{10}{c}{ Grupo 1 } & \multicolumn{10}{c}{ Grupo 2 } \\
\hline & \multicolumn{1}{c}{ Fase 1 } & \multicolumn{1}{c}{ Fase 2 } & \multicolumn{2}{c}{ Fase 1 } & Fase 2 \\
Componente & 1 & 2 & 1 & 2 & 1 & 2 & 1 & 2 \\
Programa & FR60 & FR60 & FI t s & FI t s & FR60 & FR60 & FI t s & FI t s \\
Cor do botão & Azul & Amar & Azul & Amar & Amar & Azul & Amar & Azul \\
Custo (pt) & 0 & 0 & 1 & 10 & 0 & 0 & 10 & 1 \\
\hline
\end{tabular}

Nota. Amar = amarelo; $\mathrm{pt}=$ pontos.

Fase 1: Os participantes de ambos os grupos foram expostos ao programa de reforço múltiplo FR 60 FR 60. Nos dois componentes, um smile aparecia na tela após a emissão de 60 respostas, contados do smile anterior ou do início de cada componente. Os participantes eram expostos a este programa de reforço até que a taxa de respostas das últimas quatro sessões fossem consideradas estáveis (de acordo com o critério de estabilidade de Cumming \& Schoenfeld, 1960). A estabilidade era calculada com o Stability Check, para cada componente separadamente, utilizando as quatro últimas sessões e previa uma variação máxima da taxa de respostas de $15 \%{ }^{3}$.

Para os participantes do Grupo 1, a cor do botão de respostas era azul no Componente 1 e amarela no Componente 2, e para os participantes do Grupo 2, a cor do botão de respostas era amarela no Componente 1 e azul no Componente 2.

Fase 2: Os participantes do Grupo 1 foram submetidos um múltiplo FI $t$ s-custo $1 \mathrm{FI} t$ s-custo 10 , e os participantes do Grupo 2 foram submetidos um múltiplo FI $t$ s-custo $10 \mathrm{FI} t \mathrm{~s}$-custo 1 . A ordem de apresentação do componente com custo 1 e custo 10 era a única diferença entre os participantes dos dois grupos. $\mathrm{O}$ valor do intervalo $(t)$ dos componentes foi calculado, para cada participante, com base na média dos intervalos entre reforços (IRI) de cada componente das quatro últimas sessões da Fase $1^{4}$. Durante qualquer um dos componentes, um smile aparecia na tela após a primeira resposta emitida depois da passagem de $t$ segundos desde o aparecimento do último smile ou do início de cada componente. Ao emitir a resposta de consumação, o smile desaparecia e 100 pontos eram creditados no contador. Cada resposta emitida antes de completado o intervalo de $t$ segundos era seguida da subtração de 1 ponto, no componente FIcusto 1 , ou de 10 pontos, no FI-custo 10 , no contador. As cores dos botões de respostas nos dois componentes do múltiplo eram as mesmas da Fase 1, para os dois grupos. 
Tanto na Fase 1 quanto na Fase 2, para ambos os grupos, os componentes do múltiplo alternavam-se a cada 5 minutos. A contingência de carry over não estava habilitada, ou seja, as respostas emitidas ou um intervalo de tempo transcorrido, não eram "carregados" para a próxima apresentação desse componente do múltiplo; a razão nos programas de FR e o intervalo nos FIs eram sempre reiniciados no início de cada componente. Entre um componente e outro, havia um período de 5 segundos (timeout), durante o qual os botões e o contador desapareciam, a tela ficava escura e apenas a palavra "Aguarde" era exibida em vermelho. Cada sessão experimental tinha 30 minutos de duração mais os períodos de timeout.

Ao final de cada sessão os pontos ganhos eram trocados por dinheiro ( $\mathrm{R} \$ 0,03$ para cada 100 pontos obtidos). As sessões eram realizadas de segunda a sexta-feira (entre $8 \mathrm{~h}$ e $18 \mathrm{~h}$ ), de acordo com a disponibilidade dos participantes.

\section{RESULTADOS}

A Figura 1 exibe a taxa de respostas por minuto (R/min) em cada sessão. Os gráficos dos participantes do Grupo 1 estão na coluna da esquerda e os do Grupo 2 , na coluna da direita.

Observa-se na Figura 1 que os participantes precisaram de seis a nove sessões para atingir o critério de estabilidade na Fase 1. O participante P9 (Grupo 2) foi quem mais teve variabilidade nas taxas de respostas entre todas as sessões da Fase 1. No geral, as taxas de respostas foram semelhantes entre os dois componentes do múltiplo FR 60 FR 60. As maiores diferenças nas taxas de respostas entre os dois componentes do múltiplo foram $36 \mathrm{R} / \mathrm{min}$ (Sessão 3 do participante P2) e $22 \mathrm{R} / \mathrm{min}$ (Sessão 7 do participante P9). A mediana da diferença, considerando as quatro últimas sessões de todos os participantes, foi de $2 \mathrm{R} / \mathrm{min}$. Considerando as quatro últimas sessões da Fase 1, as taxas de respostas foram sempre acima de $185 \mathrm{R} / \mathrm{min}$ para todos os participantes. 

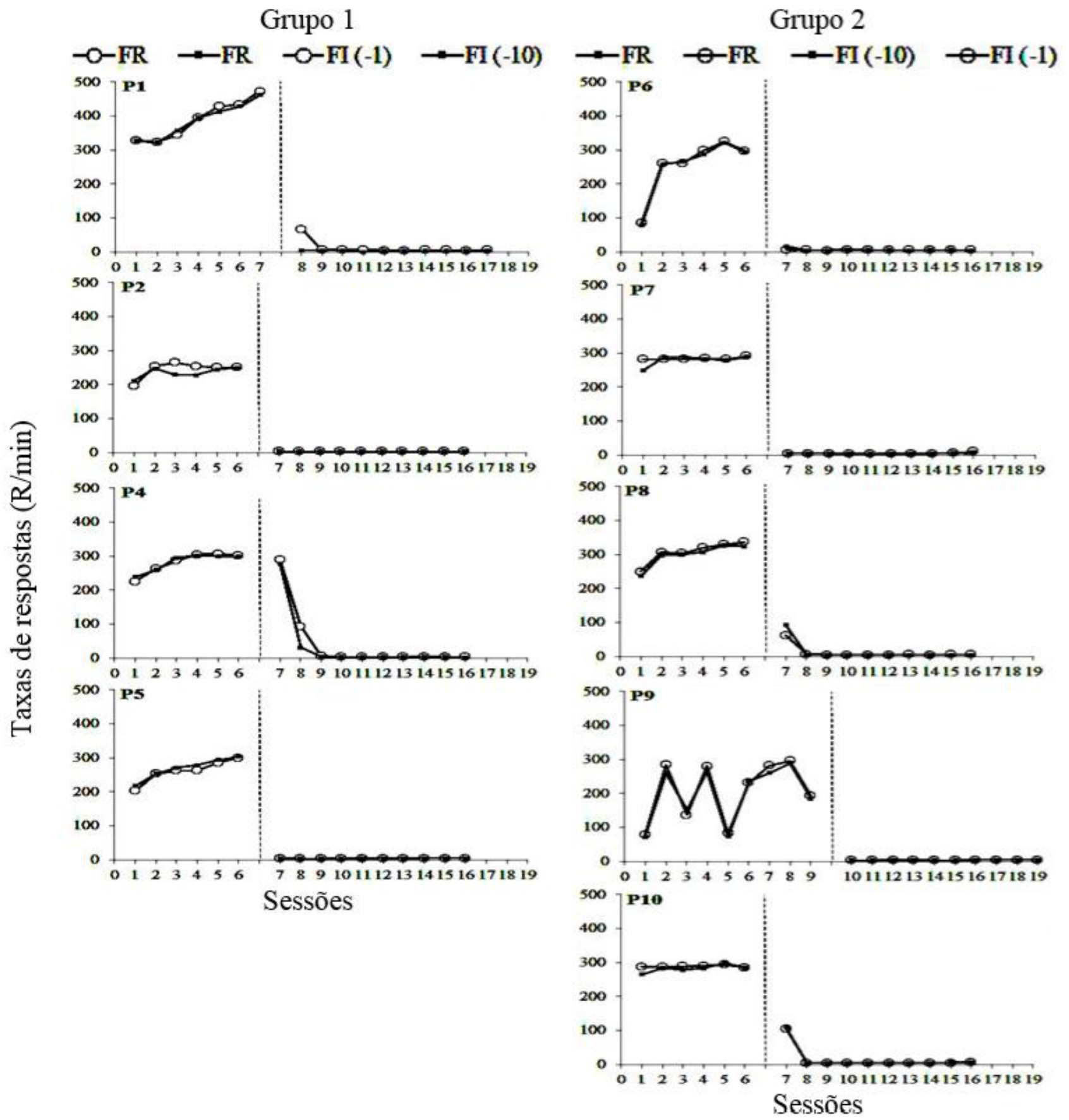

Figura 1. Taxas de respostas (R/min) por sessão dos participantes do Grupo 1 (coluna esquerda) e do Grupo 2 (coluna direita). Os pontos à esquerda da linha pontilhada referem-se a Fase 1 (múltiplo FR 60 FR 60) e os pontos à direita da linha pontilhada referem-se a Fase 2 (múltiplo FI-custo 1 FIcusto 10 para o Grupo 1 e múltiplo FI-custo $10 \mathrm{FI}$-custo 1 para o Grupo 2).

A Tabela 2 exibe, para cada participante, o Índice de Estabilidade (IE) em cada componente nas quatro últimas sessões da Fase 1; o intervalo entre reforços (IRI) médio (em segundos) das quatro últimas sessões da
Fase 1 (com o erro padrão da média); os intervalos (em segundos) dos componentes do FI na Fase 2 e o IRI médio (em segundos) em cada componente do múltiplo FI t s-custo FI t s-custo, da primeira sessão da Fase 2. 
Tabela 2

Índice de Estabilidade (IE) da taxa de respostas; média do IRI (em segundos) das quatro últimas sessões da Fase 1 ( \pm o Erro Padrão da Média); intervalo ( $t$ ) dos componentes do múltiplo Fl-custo Fl-custo na Fase 2 e o IRI médio (em segundos) da primeira sessão da Fase 2, para os participantes dos Grupos 1 e 2.

\begin{tabular}{cccccccc}
\hline & \multicolumn{4}{c}{ Fase 1 } & \multicolumn{5}{c}{ Fase 2 } \\
& \multicolumn{4}{c}{ Múltiplo FR 60 FR 60 } & \multicolumn{3}{c}{ Múltiplo FI t s-custo FI t s-custo } \\
Part. & \multicolumn{2}{c}{ IE (\%) } & \multicolumn{2}{c}{ IRI (s) } & Int. t (s) & IRI (s) \\
P1 & 9,72 & C2 & C1 & C2 & C1 e C2 & C1 & C2 \\
P2 & 3,33 & 7,78 & $8,4 \pm 0,3$ & $8,5 \pm 0,3$ & 15 & 17,5 & 17,4 \\
P4 & 2,84 & 0,17 & $12,1 \pm 0,2$ & $15,1 \pm 0,3$ & 15 & 19,4 & 19,1 \\
P5 & 10,79 & 8,38 & $12,9 \pm 0,2$ & $11,9 \pm 0,1$ & 12 & 12,0 & 12,1 \\
P6 & 10,78 & 10,49 & $12,3 \pm 0,5$ & $12,5 \pm 0,3$ & 13 & 15,7 & 15,4 \\
P7 & 0,0 & 1,40 & $12,6 \pm 0,1$ & $12,6 \pm 0,6$ & 12 & 14,4 & 13,9 \\
P8 & 6,83 & 6,67 & $11,4 \pm 0,2$ & $11,1 \pm 0,3$ & 13 & 18,7 & 15,2 \\
P9 & 4,33 & 4,79 & $15,1 \pm 1,4$ & $14,7 \pm 1,4$ & 15 & 14,0 & 14,1 \\
P10 & 3,85 & 0,00 & $12,6 \pm 0,2$ & $12,4 \pm 0,1$ & 13 & 14,0 & 21,6 \\
\hline
\end{tabular}

Nota. IE = Índice de estabilidade; IRI (s) = Intervalo entre reforços; Int. $\mathrm{t}(\mathrm{s})$ = Intervalo do Fl; Part. = participantes;

$\mathrm{C} 1=$ Componente $1 ; \mathrm{C} 2$ = Componente 2 .

Observa-se na Tabela 2 que, na Fase 1 (múltiplo FR FR), o IE das taxas de respostas em cada componente variou de 0 a $10,78 \%$ e que a média do IRI, nas quatro últimas sessões da Fase 1, variou pouco entre os componentes (a maior variação foi de $1 \mathrm{~s}$ - participante P2). Estes valores do IRI determinaram o valor do intervalo nos componentes do múltiplo FI-custo FI-custo da Fase 2 (Int. t (s)). Houve um erro de cálculo do intervalo do participante P1 e o intervalo do FI na Fase 2 foi fixado em 15 segundos quando deveria ter sido de 9 segundos. Os intervalos do FI variaram entre 12 e 15 segundos para os participantes de ambos os grupos.

Como pode ser observado na Figura 1, quando o programa de reforço foi alterado e o custo da resposta introduzido (da Fase 1 para a Fase 2), as taxas de respostas diminuíram na primeira sessão da Fase 2 para oito dos nove participantes, ficando sempre abaixo de 110 R/min. Quatro dos nove participantes (P2; P5; P7 e P9) apresentaram mudança de maior magnitude nas taxas de respostas, mantendo-as iguais ou abaixo de 5,2 R/min em ambos os componentes do múltiplo. $\mathrm{O}$ participante $\mathrm{P} 4$, que emitiu em média $299 \mathrm{R} / \mathrm{min}$ em ambos os componentes na Fase 1, manteve a taxa de respostas acima de $277 \mathrm{R} / \mathrm{min}$ na primeira sessão e iguais ou abaixo de 7,2 R/min na terceira sessão da Fase 2. Na última sessão da Fase 2, as taxas de respostas dos nove participantes, em ambos os componentes do múltiplo, foram iguais ou menores que $11,2 \mathrm{R} / \mathrm{min}$.
A estabilidade da taxa de respostas nas últimas quatro sessões da Fase 1 (ver Tabela 2) não pareceu relacionada com a persistência de altas taxas de respostas na Fase 2. Apesar de os valores do IE de P4 terem sido baixos e de as taxas de respostas desse participante terem sido altas na primeira sessão da Fase 2, outros participantes (e.g., P7 e P10) tiveram IE abaixo de $4 \%$ em ambos os componentes da Fase 1 e diminuíram as taxas de respostas na primeira sessão da Fase 2. Além disso, quando houve diferença no IE entre os componentes (P2, P4, P5, P7 e P10) tal diferença não foi preditiva dos efeitos de história observados na Fase 2.

O IRI da primeira sessão da Fase 2 (ver Tabela 2) foi maior do que o intervalo do FI para oito dos nove participantes (exceto $\mathrm{P} 4$ ). Se as taxas de respostas permanecessem altas nesta primeira sessão, como eram na última sessão da Fase 1, os IRIs seriam muito próximos aos do intervalo $(t)$ do $\mathrm{FI}$, como ocorreu com P4. Essa diferença entre o intervalo do FI e o IRI obtido, sugere que os participantes passaram a fazer pausas mais longas entre as respostas do que na fase anterior e a resposta seguida pelo smile ultrapassava, quase sempre, o intervalo do FI.

Tomados em conjunto, os dados da Figura 1 e da Tabela 2 permitem observar que (1) a taxa de respostas diminuiu quando as contingências foram alteradas de FR para FI-custo para a maioria dos participantes logo na primeira sessão da transição; (2) a estabilidade 
das taxas de respostas (medida pelo IE) não esteve correlacionada com a probabilidade de persistência comportamental na contingência de FI-custo; (3) apesar da tentativa de controlar o IRI, o comportamento dos participantes alterou-se de tal maneira (com pausas que excediam o IRI programado) que o IRI entre a Fase 1 e a Fase 2 foi diferente na maioria dos casos.

A Figura 1 permite observar que a taxa de respostas diminuiu quando o programa de reforço mudou, mas as Figuras 2 e 3 permitem observar, de maneira mais molecular, as taxas de respostas na transição da Fase 1 para a Fase 2. As Figuras 2 e 3 exibem os registros cumulativos da última apresentação de cada componente da última sessão do múltiplo FR FR (primeira linha de cada painel) e de cada componente da primeira sessão do múltiplo FI-custo FI-custo (segunda, terceira e quarta linha em cada painel) dos participantes do Grupo 1 (Figura 2) e do Grupo 2 (Figura 3). Os registros cumulativos de cada participante foram "recortados" em períodos de 5 minutos (a duração de cada componente), de modo que os registros da esquerda, de cada participante, referem-se aos períodos do Componente 1 e os registros da direita referem-se aos períodos do Componente 2. A sequência dos registros para cada participante deve ser lida a partir da primeira linha da coluna esquerda e terminar na quarta linha da coluna da direita. As marcas diagonais nos registros referem-se ao aparecimento do smile. As curvas de respostas retornavam a zero após 250 respostas.

Observa-se nas Figuras 2 e 3 que a taxa de respostas nos últimos 10 minutos do múltiplo FR FR foi de taxas altas e relativamente constantes (i.e., com poucas e curtas pausas) para os participantes de ambos os grupos. O desempenho final na Fase 1, exibido nas Figuras 2 e 3, é representativo do desempenho dos participantes nas quatro sessões finais dessa fase. Os participantes P2 e P5 (Grupo 1) e P7 e P9 (Grupo 2) diminuíram a taxa de respostas no início da primeira apresentação do múltiplo FI-custo FI-custo. Os participantes P8 e P10 responderam em taxas altas na primeira apresentação do componente de FI-custo $10 \mathrm{e}$ FI-custo 1 e, a partir da segunda apresentação de cada componente, a taxa de respostas diminuiu para esses dois participantes. Os participantes P1 e P6 mantiveram taxas de respostas altas no início da primeira sessão da Fase 2, mas diminuíram a taxa de respostas antes do final da apresentação do primeiro componente do múltiplo (FI-custo 1, para P1, e FI-custo 10, para P6). Apenas P4 manteve taxas de respostas altas durante toda a primeira sessão da Fase 2. Todos os participantes (exceto P4) emitiram baixas taxas de respostas nas últimas apresentações de cada componente do múltiplo FI-custo FI-custo e o padrão de respostas, nos dois últimos componentes da primeira sessão da Fase 2, foi o mesmo observado na última sessão de exposição ao múltiplo FI-custo FI-custo (dados não exibidos).

Os registros cumulativos da transição do múltiplo FR FR para o múltiplo FI-custo FI-custo (Figuras 2 e 3 ) indicam que as taxas de respostas diminuíram e o padrão temporal das respostas também mudou. Isso permite afirmar que a distribuição temporal das respostas no final primeira sessão da Fase 2, não foi uma "herança" do padrão observado na Fase 1 (exceto, talvez, para P4). 


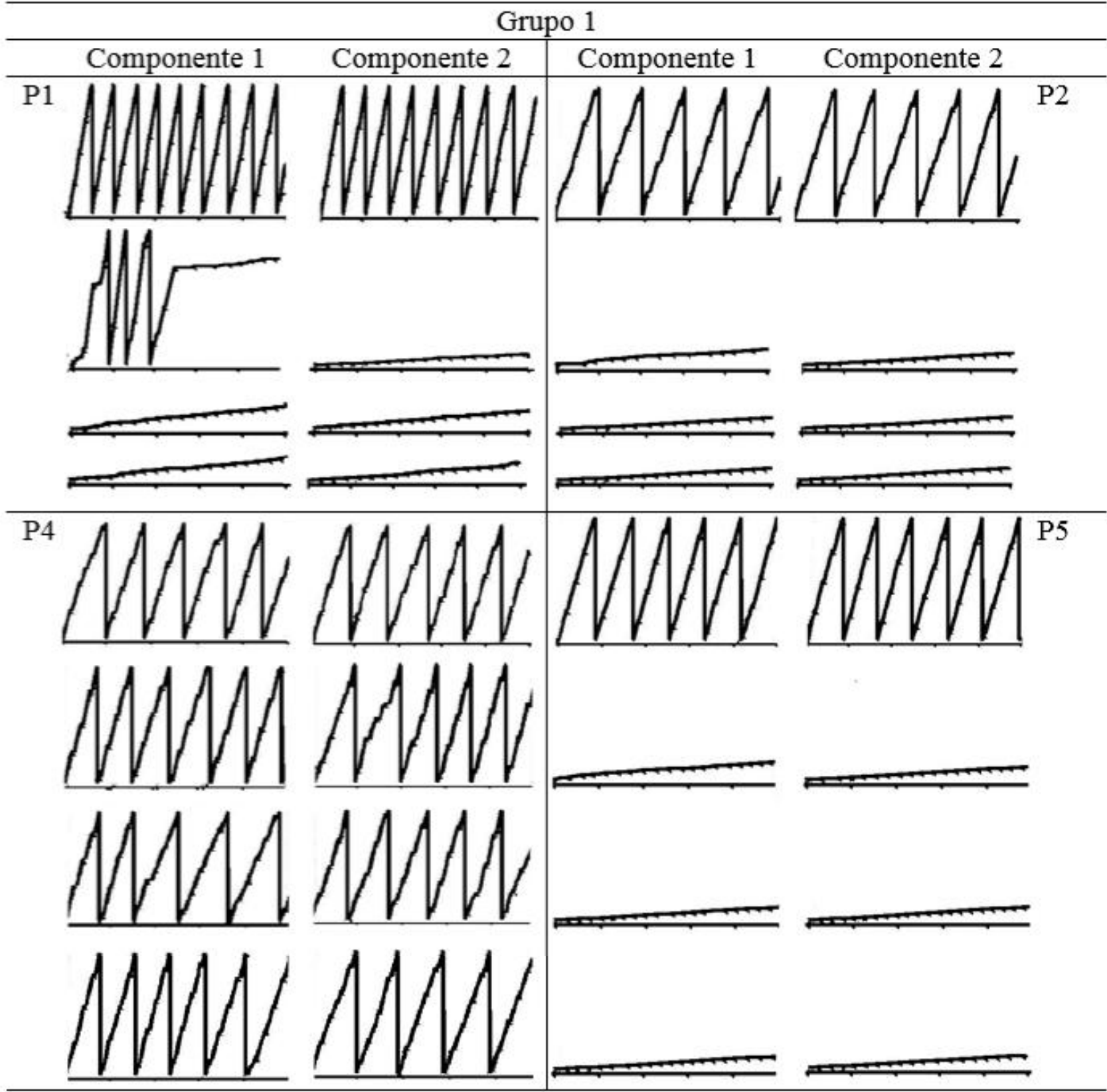

Figura 2. Registros cumulativos da última apresentação de cada componente da última sessão do múltiplo FR FR (primeira linha de cada participante) e de cada componente (em períodos de 5 minutos) da primeira sessão do múltiplo FI-custo Fl-custo dos participantes do Grupo 1. As marcas diagonais nos registros referem-se ao aparecimento do smile. As curvas de respostas retornavam a zero após 250 respostas. (Mais detalhes no texto.) 


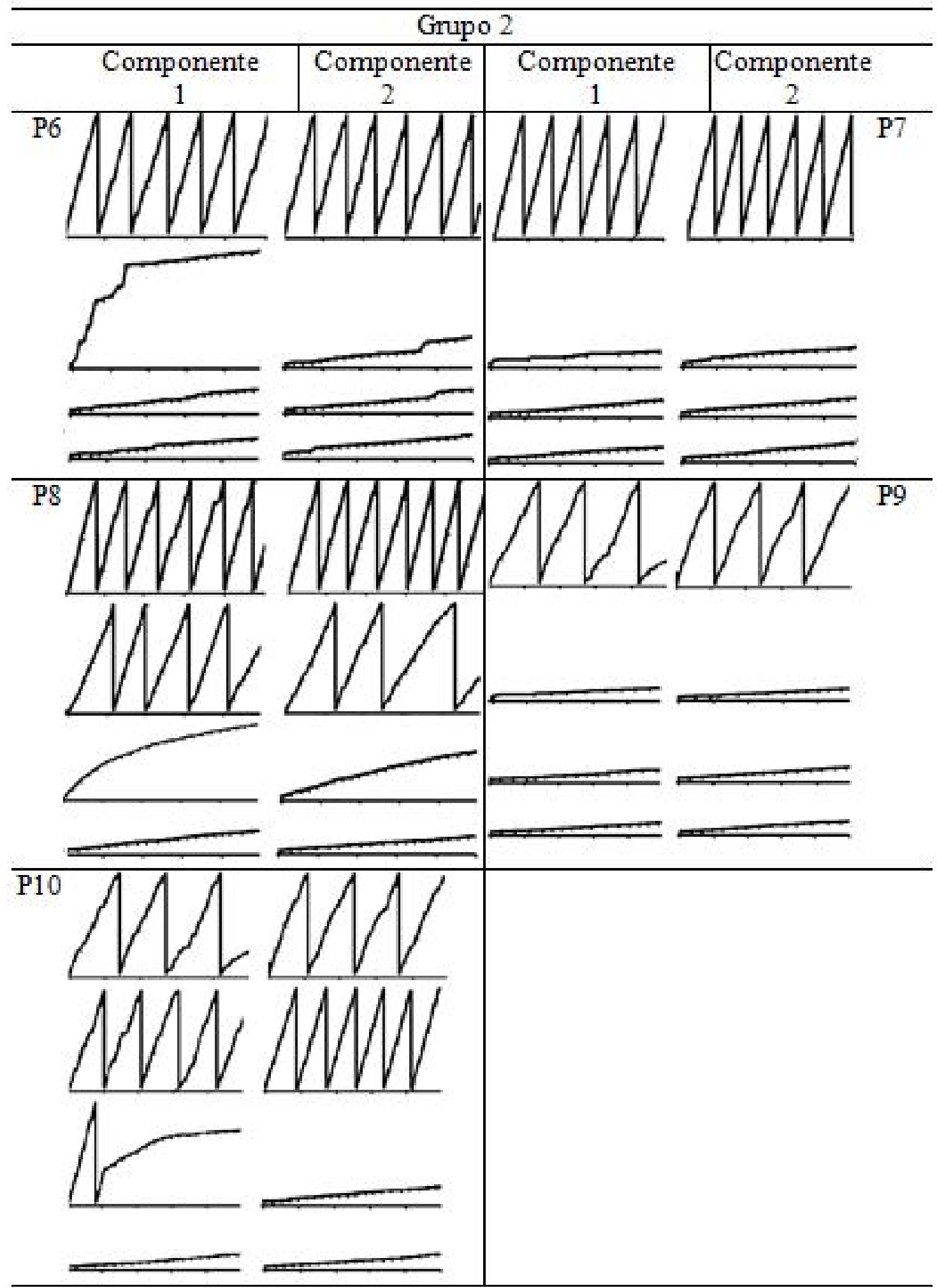

Figura 3. Registros cumulativos da última apresentação de cada componente da última sessão do múltiplo FR FR (primeira linha de cada participante) e de cada componente da primeira sessão do múltiplo Fl-custo Fl-custo dos participantes do Grupo 2. (Mais detalhes na legenda da Figura 2 e no texto.) 
A Figura 4 permite comparar os últimos 10 minutos da última sessão da Fase 1 com os últimos 10 minutos da primeira sessão da Fase 2 no que se refere a distribuição das respostas dentro dos IRIs, com o intuito de avaliar se o comportamento dos participantes estava sob o controle do parâmetro temporal da contingência de FI. Essa Figura exibe como as respostas emitidas nos dois últimos componentes da última sessão da Fase 1 (múltiplo FR FR; lado esquerdo da linha tracejada em cada gráfico) e nos dois últimos componentes da primeira sessão da Fase 2 (múltiplo FI-custo FI-custo; lado direito da linha tracejada em cada gráfico) foram distribuídas dentro dos IRIs, em blocos de 3 s. O intervalo $0,0-2,9$ no eixo $x$ de cada gráfico exibe a porcentagem de respostas dos 10 minutos finais de cada sessão que foram emitidas dentro dos intervalos de 1 décimo de segundo a 2,9 segundos dos IRIs; o intervalo 3,0-5,9 exibe a porcentagem de respostas da sessão que foram emitidas dentro dos intervalos de 3 a 5,9 segundos dos IRIs e assim por diante. Quando o intervalo do FI foi de 13 segundos (P5, P7 e P10), o último bloco contém a porcentagem de respostas que foram emitidas após a passagem de 12 segundos do intervalo. As barras vazias referem-se ao Componente 1 (FR para ambos os grupos no lado esquerdo da linha tracejada e FI-custo 1 para o Grupo 1 e FI-custo 10 para o Grupo 2 do lado direito da linha tracejada) e as barras cheias referem-se ao Componente 2 (FR para ambos os grupos no lado esquerdo da linha tracejada e FI-custo 10 para o Grupo 1 e FI-custo 1 para o Grupo 2 do lado direito da linha tracejada).

Observa-se na Figura 4 que a distribuição das respostas durante o final da última sessão da Fase 1 (múltiplo FR FR) foi relativamente constante nos dois componentes entre os blocos de intervalos para todos os participantes (ver também primeira linha de cada participante das Figuras 2 e 3). As respostas de P1 terminaram no terceiro bloco porque, como apontado anteriormente (ver Tabela 2), o IRI do múltiplo FR FR era de 8,5 s em média. Nos 10 minutos finais da primeira sessão da Fase 2 (múltiplo FI-custo FI-custo), a distribuição das respostas ao longo do intervalo do FI parece ter ficado sob o controle do parâmetro temporal do intervalo para oito dos nove participantes (exceto P4 - ver também Figuras 1 e 2). Nesses casos, a maioria das respostas, no final da primeira sessão da Fase 2, foi emitida no final ou depois de completado o intervalo do FI (penúltimo e último bloco de intervalo). A distribuição das respostas ao longo do intervalo do FI, não pareceu correlacionada com o valor do custo (custo 1 vs. custo 10), nem com a ordem de apresentação do custo (Grupo 1 vs. Grupo 2).

Tomados em conjunto os resultados das Figuras 2, 3 e 4 indicam que, não apenas a taxa de respostas diminuiu para oito de nove participantes logo na primeira sessão de transição da Fase 1 para a Fase 2, mas que a distribuição temporal das respostas também alterou-se, o que sugere que o comportamento da maioria dos participantes ficou sob o controle do parâmetro temporal da contingência do FI no final da primeira sessão do múltiplo FI-custo FI-custo.

A resistência do comportamento à mudança foi avaliada pela proporção de mudança da taxa de respostas entre a Fase 1 e a primeira sessão da Fase 2 (cf. Nevin, 1974, 1979). A Figura 5 exibe a proporção de mudança da taxa de respostas (em escala logarítmica de base 10) na primeira sessão do múltiplo FI-custo FI-custo em relação à média da taxa de respostas das quatro últimas sessões do múltiplo FR FR (linha de base, LB), para cada participante do experimento. Os gráficos na coluna esquerda são dos participantes do Grupo 1 e os da coluna direita são dos participantes do Grupo 2. A proporção foi calculada dividindo-se a média da taxa de respostas $(\mathrm{R} / \mathrm{min})$ em blocos de 5 minutos, de cada componente, da primeira sessão do múltiplo FI-custo FI-custo, na Fase 2 pela média da taxa de respostas (R/min) das quatro últimas sessões da Fase 1 (em cada componente de FR, separadamente). Quanto mais próximo qualquer ponto dos gráficos estiver do valor 1 do eixo $y$, menor foi a variação das taxas de respostas na Fase 2 em relação à LB. O primeiro gráfico de cada coluna exibe o mesmo cálculo, mas tomando a média de todos os participantes em cada grupo.

No Grupo 1, o participante P1 exibiu um desempenho no FI-custo 1 (Componente 1 para o Grupo 1) mais resistente à mudança do que no FI-custo 10 (Componente 2 para o Grupo 1) e o mesmo efeito, porém com menor magnitude, foi observado no comportamento de P2, P4 e P5. No Grupo 2, praticamente não houve diferença na resistência do comportamento à mudança nos dois componentes de FI-custo para P7 e P9. Para P8, o comportamento no FI-custo 10 (Componente 1 para o Grupo 2) foi mais resistente à mudança do que no FI-custo 1 (Componente 2 para o Grupo 2). Essa diferença entre os componentes em relação à resistência do comportamento à mudança apareceu em um dos blocos de cinco minutos para P6 e P10 (primeiro e segundo bloco de cinco minutos, respectivamente); em ambos os casos o FI-custo 10 foi mais resistente à mudança do que o FI-custo 1 . 


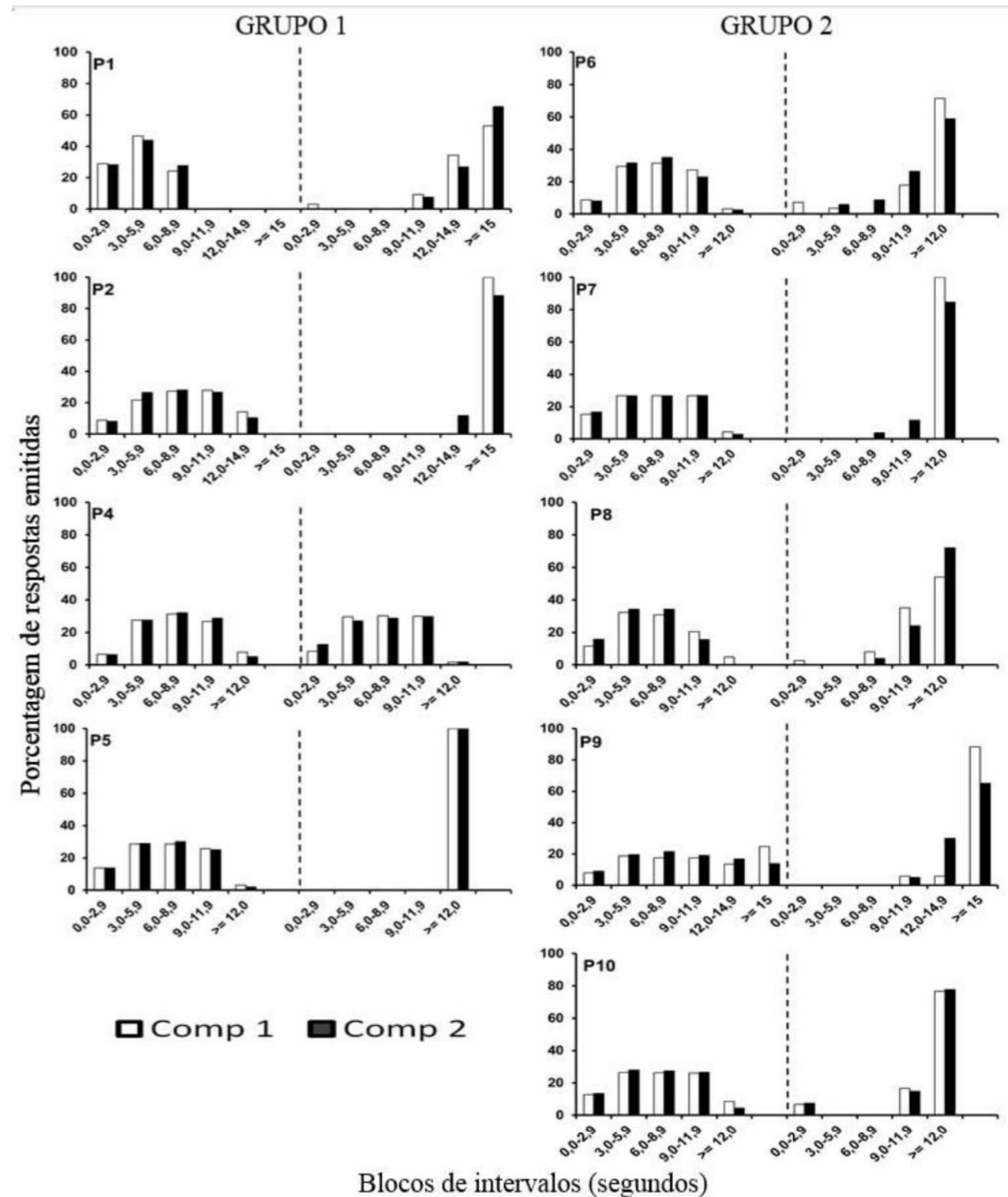

Figura 4. Porcentagem de respostas emitidas nos IRIs nos dois últimos componentes da última sessão da Fase 1 (múltiplo FR FR; lado esquerdo da linha tracejada em cada gráfico) e nos dois últimos componentes da primeira sessão da Fase 2 (múltiplo FI-custo FI-custo; lado direito da linha tracejada em cada gráfico) em blocos sucessivos de 3 segundos. As barras vazias referem-se ao Componente 1 e as barras ao Componente 2. (Mais detalhes no texto.) 

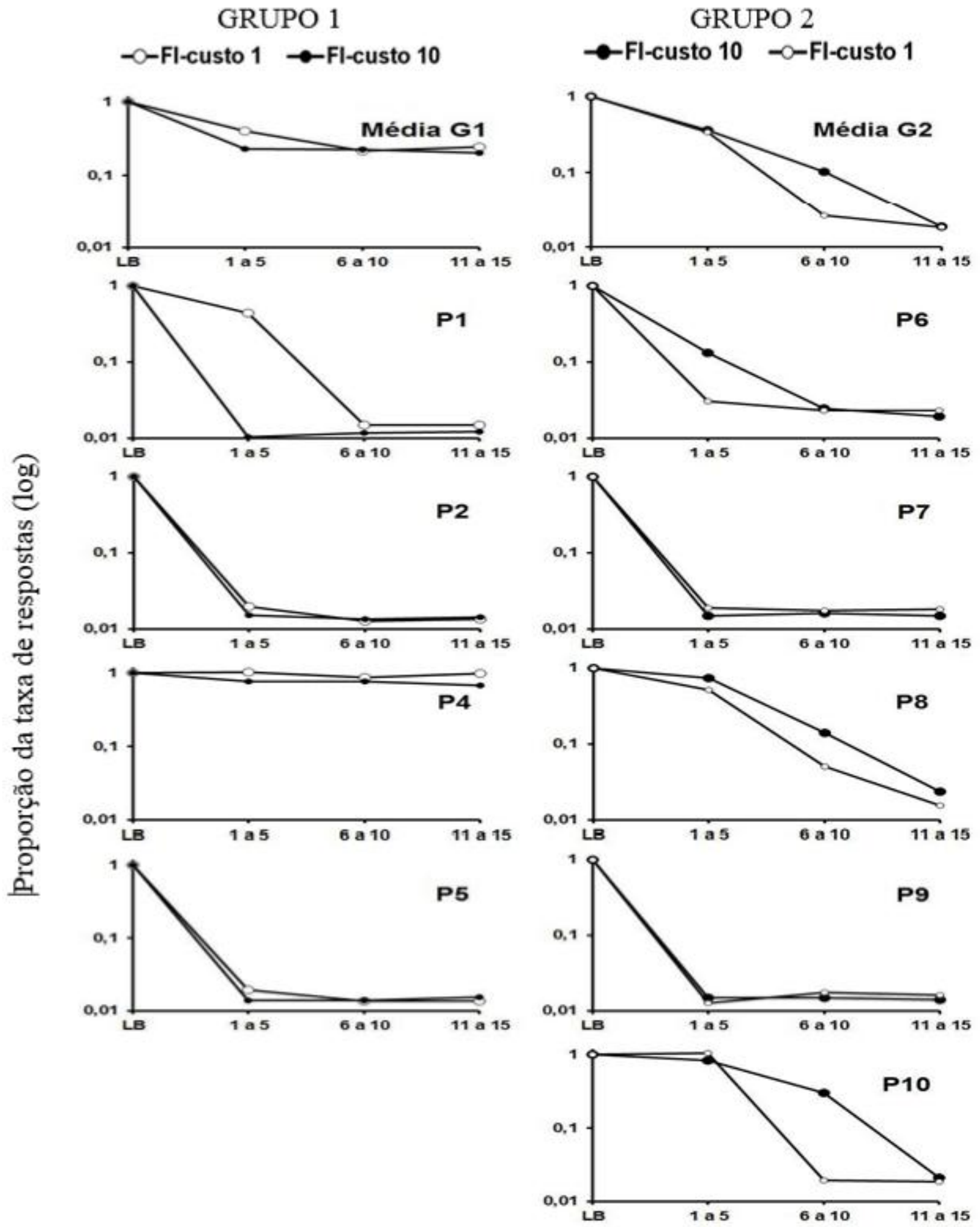

Blocos de intervalo ( $\mathrm{min}$ )

Figura 5. Log da proporção de mudança da taxa de respostas no Fl-custo1 (círculos vazios) e no Fl-custo 10 (círculos cheios) para cada participante. A proporção de mudança foi calculada a partir da taxa média de respostas $(\mathrm{R} / \mathrm{min})$ em blocos de cinco minutos da primeira sessão da Fase 2 , relativa à média da taxa de respostas(R/min) nas quatro últimas sessões do múltiplo FR FR, da Fase 1 (LB). 
Em geral, para ambos os grupos, tanto no componente com custo 1 quanto no componente com custo 10 , a proporção de mudança na taxa de respostas em relação à $\mathrm{LB}$ foi alta e relativamente rápida. Apenas P4 exibiu maior persistência comportamental durante toda a primeira sessão de exposição ao múltiplo FIcusto FI-custo e P8 e P10 no primeiro terço da sessão de transição. Para os participantes do Grupo 1 o componente com custo 1 foi, no geral, mais resistente a mudança enquanto para os participantes do Grupo $2 \mathrm{o}$ componente com custo 10 pareceu mais resistente à mudança (ver os gráficos com a média de cada grupo na Figura 5), sugerindo um efeito de ordem de apresentação da magnitude do custo, sobre a velocidade com que a taxa de respostas mudou na Fase 2 (i.e., o Componente 1 , na média, mais resistente à mudança do que o Componente 2, independentemente da magnitude o custo programado).

\section{DISCUSSÃO}

Os resultados deste experimento corroboraram aqueles obtidos por Costa et al. (2009). A mudança da contingência de razão para uma contingência de FI com custo diminuiu a taxa de respostas para os nove participantes - independentemente da contingência de FI com custo 1 ter precedido ou sucedido a contingência de FI com custo 10. Esses resultados são semelhantes aos de alguns experimentos (e.g., Chung, 1965, Experimento I; Miller, 1968; Mowrer \& Jones, 1943; Skinner \& Morse, 1958) que sugerem que a introdução (ou aumento) do custo da resposta tende a diminuir a taxa de respostas. Todavia, esses estudos manipularam apenas o custo da resposta, enquanto no presente estudo e outros (e.g., Costa et al.; Weiner, 1965, 1969, 1970) além da introdução do custo (perda de pontos) a contingência de reforço foi alterada (de FR para FI).

Maior resistência do comportamento à mudança, quando houve (e.g., P1, P6, P8 e P10 - ver também o gráfico com a média dos grupos no topo da Figura 5), pareceu mais relacionada à ordem de apresentação do componente do que ao valor do custo da resposta em si (i.e., se a sessão começava com o FI-custo 1 - Grupo 1 , ou se a sessão começava com o FI-custo 10 Grupo 2). Com isso, os resultados do presente estudo sugerem que a maior resistência à mudança observada no FI-custo 1, em relação ao FI-custo 10 , do estudo de Costa et al. (2009) pode ter resultado da ordem de exposição à contingência de FI-custo (naquele estudo a exposição ao FI-custo 1 sempre precedeu a exposição ao FI-custo 10).

Efeito da história de exposição ao FR foi observado, por mais de uma sessão, apenas para o participante P4 (ver Figura 1). Para oito dos nove participantes (exceto P4) a taxa de respostas nos 10 minutos finais da primeira sessão da Fase 2 (ver Figuras 2 e 3, quarta linha de cada participante) havia diminuído a um valor que foi mantido relativamente constante até a última sessão do estudo e a distribuição das respostas no final da Fase 1 e na primeira sessão da transição sugere que o comportamento ficou sob o controle do parâmetro temporal do FI para esses oito participantes (ver Figura 4).

Os resultados do presente estudo, assim como os de Costa et al. (2009), foram diferentes dos obtidos por Weiner $(1965,1969,1970)$, que observou persistência comportamental (manutenção de taxas de respostas relativamente altas) para a maioria dos participantes em FI-custo após uma história de exposição a contingências de razão. No estudo de Costa et al., os participantes haviam sido expostos ao FR por três sessões de 15 minutos (45 min), enquanto que nos estudos de Weiner esta exposição foi de 10 sessões de 1 hora (10 h). No presente estudo a exposição ao múltiplo FR FR foi realizada até que o comportamento atingisse um critério de estabilidade; os participantes foram expostos a, no mínimo, seis sessões de $30 \mathrm{mi}-$ nutos (3h). Apesar do maior tempo de exposição ao FR, quando comparado com a pesquisa de Costa et al., e da adoção do critério de estabilidade das taxas de respostas, ainda é possível questionar se um maior tempo de exposição à contingência de FR poderia produzir maior persistência comportamental (cf. Lattal \& Neef, 1996) em função da maior quantidade de reforço que uma história mais extensa de responder em FR poderia proporcionar (cf. Nevin, 1974; 1979). Pesquisas futuras deveriam testar diretamente o efeito da duração da fase de construção da história sobre a persistência comportamental em uma fase teste subsequente.

Joyce e Chase (1990) sugeriram que a probabilidade de mudança do comportamento pode ser afetada pela maior ou menor estabilidade da linha de base. A diferença nos resultados da persistência comportamental entre os estudos de Weiner (1965, 1969 e 1970) e o presente estudo poderia estar relacionada à diferença na estabilidade das taxas de respostas? Aparentemente não. A estabilidade da taxa de respostas nas últimas quatro sessões da Fase 1 (ver Tabela 2) 
não pareceu relacionada à probabilidade de maior ou menor persistência de taxas de respostas relativamente altas na Fase 2. No entanto, é importante observar que no presente estudo a variabilidade das taxas de respostas na linha de base foi restrita à faixa permitida pelo critério de estabilidade adotado. A análise visual da estabilidade da taxa de respostas dos participantes do estudo de Weiner (1969, Experimento 2, Figuras 5 e 7) sugerem que participantes do estudo de Weiner apresentaram estabilidade das quatro últimas sessões em FR comparáveis com a estabilidade encontrada para a maioria dos participantes do presente estudo nas sessões finais da Fase 1 (Figura 1) e, enquanto as altas taxas de respostas emitidas pelos participantes de Weiner persistiram durante o FI (com e sem custo) por 10 sessões de $1 \mathrm{~h}$ cada, no presente estudo a persistência, quando existiu, foi de curta duração.

Diferentemente da pesquisa de Costa et al. (2009), que analisou, mas não controlou diretamente o IRI entre as fases do estudo, a presente pesquisa procurou controlar o IRI entre as duas fases do experimento (ver Tabela 2). Isso foi feito porque segundo Okouchi (2003, 2007, 2010), o IRI pode exercer uma função discriminativa no comportamento de humanos. Os resultados da presente pesquisa apontaram que o IRI não foi bem controlado. Isso ocorreu porque, embora o intervalo do FI tenha sido determinado pelo IRI da fase anterior, os participantes começaram a fazer pausas que excediam o valor do intervalo do FI. Apesar disso, é interessante observar que, o participante P1, cujo IRI foi bastante discrepante entre as Fases 1 e 2 do experimento, demorou mais do que outros participantes (e.g., $\mathrm{P} 2, \mathrm{P} 6, \mathrm{P} 7, \mathrm{P} 5, \mathrm{P} 9$, cujo valor do intervalo do FI foi mais próximo do IRI da Fase 1 do experimento) para alterar a taxa de respostas quando a contingência mudou de FR para FI custo (ver os registros cumulativos nas Figuras 2 e 3). Estudos posteriores poderiam manipular diretamente o IRI (cf. Okouchi, 2003) para verificar o papel dessa variável sobre os efeitos da história de responder em FR sobre o desempenho subsequente em FI-custo.

Tomados em conjunto, os resultados do presente estudo sugerem que a mudança da contingência de reforço de um múltiplo FR FR para um múltiplo FIcusto FI-custo aumenta a probabilidade de diminuição na taxa de respostas (i.e., as altas taxas de respostas selecionadas durante a exposição ao FR diminuem quando o FI-custo é implementado) e que, nos valores empregados no presente estudo, a magnitude do custo não teve efeitos diferenciais. Diferentemente dos estudos de Weiner $(1965,1969 ; 1970)$, os resultados do presente estudo e os de Costa et al. (2009) indicaram que a implementação de um FI-custo 1, após uma história de FR foi suficiente para que as altas taxas de respostas selecionadas durante o FR diminuíssem. Como as taxas de respostas tenderam a cair para próximo de uma resposta por intervalo no FI-custo 1, o aumento do custo para 10 não poderia produzir uma redução ainda maior na taxa de respostas sem que isso implicasse na diminuição na quantidade de pontos obtidos.

\section{REFERÊNCIAS}

Azrin, N. H. (1960). Effects of punishment intensity during variable-interval reinforcement. Journal of the Experimental Analysis of Behavior, 3, 123-142.

Azrin, N. H., \& Holz, W. C. (1961). Punishment during fixedinterval reinforcement. Journal of the Experimental Analysis of Behavior, 4, 343-347.

Chung, S.-H. (1965). Effects of effort on response rate. Journal of the Experimental Analysis of Behavior, 8, 1-7.

Costa, C. E., \& Banaco, R. A. (2002). ProgRef v3: Sistema computadorizado para coleta de dados sobre programas de reforço com humanos - Recursos básicos. Revista Brasileira de Terapia Comportamental e Cognitiva, 4, 173-192.

Costa, C. E., \& Banaco, R. A. (2003). ProgRef v3: Sistema computadorizado para coleta de dados sobre programas de reforço com humanos - Recursos adicionais. Revista Brasileira de Terapia Comportamental e Cognitiva, 5, 219-229.

Costa, C. E., \& Cançado, C. R. X. (2012). Stability Check: A program for calculating the stability of behavior. Mexican Journal of Behavior Analysis, 38, 61-71.

Costa, C. E., Cirino, S. D., Cançado, C. R. X., \& Soares, P. G. (2009). Polêmicas sobre história comportamental: Identificação de seus efeitos e sua duração. Psicologia: Reflexão e Crítica, 22, 394-403.

Costa, C. E., Soares, P. G., Becker, R. M., \& Banaco, R. A. (2009). O efeito da magnitude do custo da resposta e do evento consequente empregado sobre o comportamento em FI após uma história de FR. Revista Brasileira de Análise do Comportamento, 5, 89-106.

Freeman, T. J., \& Lattal, K. A. (1992). Stimulus control of behavioral history. Journal of the Experimental Analysis of Behavior, 57, 5-15.

Joyce, J. H., \& Chase, P. N. (1990). Effects of response variability on the sensitivity of rule-governed behavior. Journal of the Experimental Analysis of Behavior, 54, 251-262.

Lattal, K. A., \& Neef, N. A. (1996). Recent reinforcementschedule research and applied behavior analysis. Journal of Applied Behavior Analysis, 29, 213-230.

Matthews, B. A., Shimoff, E., Catania, A. C., \& Sagvolden, T. (1977). Uninstructed human responding: Sensitivity to ratio and interval contingencies. Journal of the Experimental Analysis of Behavior, 27, 453-467. 
Miller, L. K. (1968). Escape from an effortful situation. Journal of the Experimental Analysis of Behavior, 11, 619-627.

Mowrer, O. H., \& Jones, H. M. (1943). Extinction and behavior variability as functions of effortfulness of task. Journal of Experimental Psychology, 33, 369-386.

Nevin, J. A. (1974). Response strenght in multiple schedules. Journal of the Experimental Analysis of Behavior, 21, 389-408.

Nevin, J. A. (1979). Reinforcement schedules and response strength. In M. Zeiler \& P. Harzem (Eds.), Reinforcement and organization of behavior (pp. 117-158). New York: John Wiley \& Sons.

Okouchi, H. (2003). Effects of differences in interreinforcer intervals between past and current schedules on fixed-interval responding. Journal of the Experimental Analysis of Behavior, 79, 49-64.

Okouchi, H. (2007). An exploration of remote history effects in humans. Psychological Record, 57, 241-263.

Okouchi, H. (2010). An exploration of remote history effects in humans: II. The effects under fixed-interval, variable-interval, and fixed-ratio schedules. Psychological Record, 60, 27-42.
Skinner, B. F., \& Morse, W. H. (1958). Fixed-interval reinforcement of running in a wheel. Journal of the Experimental Analysis of Behavior, 1, 371-379.

Todorov, J. C. (1971). Concurrent performances: Effect of punishment contingent on the switching response. Journal of the Experimental Analysis of Behavior, 16, 51-62.

Wanchisen, B. A. (1990). Forgetting the lessons of history. Behavior Analyst, 13, 31-37.

Weiner, H. (1965). Conditioning history and maladaptative human operant behavior. Psychological Reports, 17, 935-942.

Weiner, H. (1969). Controlling human fixed-interval performance. Journal of the Experimental Analysis of Behavior, 12, 349-373.

Weiner, H. (1970). Human behavioral persistence. Psychological Record, 20, 445-456.

Recebido: $21 / 04 / 2012$

Última Revisão: $16 / 11 / 2012$

Aceito: $27 / 11 / 2012$

Notas:

1 Esta pesquisa foi parcialmente financiada com recursos da Fundação Araucária (Programa de Apoio a Pesquisa Básica e Aplicada Chamada 14/2009 - protocolo 10924). Os autores agradecem ao Dr. Carlos Renato Xavier Cançado pela revisão e sugestões feitas para este artigo. João P. P. de Souza e Thais Bianchini foram Bolsistas IC/UEL durante a coleta de dados deste trabalho.

2 O número exato de sessões era difícil de precisar porque dependia do desempenho do participante para atingir a estabilidade. Os pesquisadores, baseados em sua experiência, avaliaram que 20 sessões seriam suficientes para completar o estudo. Por isso foi informado este número de sessões ao participante, embora - como pode ser visto na seção de Resultados - o número de sessões para todos os participantes foi menor que 20 .

3 A fórmula para o cálculo da estabilidade era:

$$
\text { I.E. }=\left|\frac{\left[\left(\frac{S 1+S 2}{2}\right)-\left(\frac{S 3+S 4}{2}\right)\right]}{\left(\frac{S 1+S 2+S 3+S 4}{4}\right)}\right| \times 100
$$

Onde,

I.E. = índice de estabilidade;

$\mathrm{Sn}=$ taxa de respostas na sessão $\mathrm{n}$.

4 Para esse cálculo foram tomados os IRIs "válidos". Como o carry over não estava habilitado (ver descrição sobre isso adiante no texto) ocorriam respostas que não eram reforçadas no final de cada apresentação de cada componente. Essas respostas - e o tempo gasto em emiti-las - foram ignoradas para o cálculo do IRI, pois no início de cada componente um "novo" IRI era iniciado. Em resumo, os IRIs "válido" em cada componente consistiram de todo IRI contado do início de um componente até o último smile liberado dentro daquele componente. 